\title{
Elementos de la cadena de valor que inciden en el desarrollo de la actividad turística del Parque Nacional Yasuní.
}

\author{
Elements of the value chain that affect the development of tourism activities \\ in Yasuní National Park
}

Sandra Ximena Valdez Quinte. ${ }^{1}$, Edison Oliver Segura Chávez. ${ }^{2}$, Enrique Armando Cabanilla Vásconez. ${ }^{3}$ \& Edison Eduardo Ruiz Mármol. ${ }^{4}$

\begin{abstract}
.
DOI: https://doi.org/10.33262/concienciadigital.v4i2.2.1782

In the current economic scenario, tourism emerges as one of the most dynamic activities of global capitalism. It is a fundamental economic activity for the generation of foreign exchange, investments, jobs and activation of productive chains. The objective of the research focused on identifying the elements that make up the value chain and that affect tourism activity in Yasuní National Park. In the methodological process, exploratory and descriptive research was applied to the available information; the variables identified from the interviews conducted with the protected area's tourism stakeholders were processed through statistical analysis using the principal components technique, whose result expressed with $63.89 \%$ of variance explains the system with four components, which led to the structuring of a map that reflects the Yasuní National Park's value chain. Finally, it shows the interrelationships and linkages between service providers in the chain; the map is the core of any further analysis of information needs, interventions and strategic collaboration to achieve long-term objectives.
\end{abstract}

\footnotetext{
${ }^{1}$ Universidad Estatal Amazónica, Maestrante Centro de Posgrados, Pastaza, Ecuador, sx.valdezq@uea.edu.ec, https://orcid.org/0000-0001-9951-2062

${ }^{2}$ Universidad Estatal Amazónica, Centro de Posgrado, Pastaza, Ecuador, esegura@uea.edu.ec, https://orcid.org/0000-0002-5274-8825

${ }^{3}$ Universidad Central del Ecuador, Facultad de Ciencias Agrícolas, Pichincha, Ecuador, eacabanilla@uce.edu.ec, https://orcid.org/0000-0002-2853-291

${ }^{4}$ Universidad Estatal Amazónica, Facultad de Ciencias de la Vida, Pastaza, Ecuador. eruiz@uea.edu.ec, https://orcid.org/0000-0001-8968-8814
} 
Keywords: Multivariate analysis, protected area, components,

\section{Resumen}

Introducción. En el escenario económico actual, el turismo emerge como una de las actividades más dinámicas del capitalismo global. Constituye una actividad económica fundamental, por la generación de divisas, inversiones, empleos y activación de encadenamientos productivos Objetivo. Identificar los elementos que conforman la cadena de valor e inciden de manera directa en la actividad turística del Parque Nacional Yasuní. Metodología. En el proceso metodológico se aplicó la investigación exploratoria y descriptiva, frente a la información disponible; las variables identificadas a partir de las entrevistas realizadas a los actores de operación turística del área protegida fueron procesadas mediante análisis estadístico con la técnica componentes principales. Resultados. El resultado expresado con el $63.89 \%$ de varianza explica el sistema con cuatro componentes, mismos que condujeron a la estructuración visual del sistema de la cadena de valor del Parque Nacional Yasuní. Conclusión. Finalmente se evidencian las interrelaciones y los vínculos entre los prestadores de servicios de la cadena; el mapa es núcleo de cualquier análisis adicional, sobre las necesidades de información, las intervenciones, así como la colaboración estratégica para alcanzar objetivos a largo plazo.

Palabras claves: Análisis multivariado, área protegida, componentes.

\section{Introducción.}

El turismo es una industria que incorpora un amplio espectro de actividades económicas, culturales y recreativas complementándose con una serie de actividades productivas relacionadas, genera oportunidades de empleo, y permite la articulación de las cadenas de valor. Estructuralmente, aporta efectivamente en la redistribución del ingreso y mejora la calidad de vida de la población de las economías locales (MINTUR, 2019,p.9).

Hablar de cadena de valor implica partir de una clásica definición como la planteada por Porter (1985) una herramienta de gestión que permite describir el desarrollo de las actividades de valor y margen de una organización empresarial. La teoría de la Cadena de Valor también precisa que una empresa tiene una ventaja competitiva cuando es capaz de ser rentable aumentando su margen (ganancia), ya sea bajando los costos o incrementando sus ventas, es el valor que una empresa es capaz de crear para sus clientes. Es esencialmente una forma de análisis de la actividad empresarial mediante la cual se descompone una empresa en sus partes constitutivas (Figura 1), buscando identificar fuentes de ventaja competitiva en aquellas actividades generadoras de valor. 


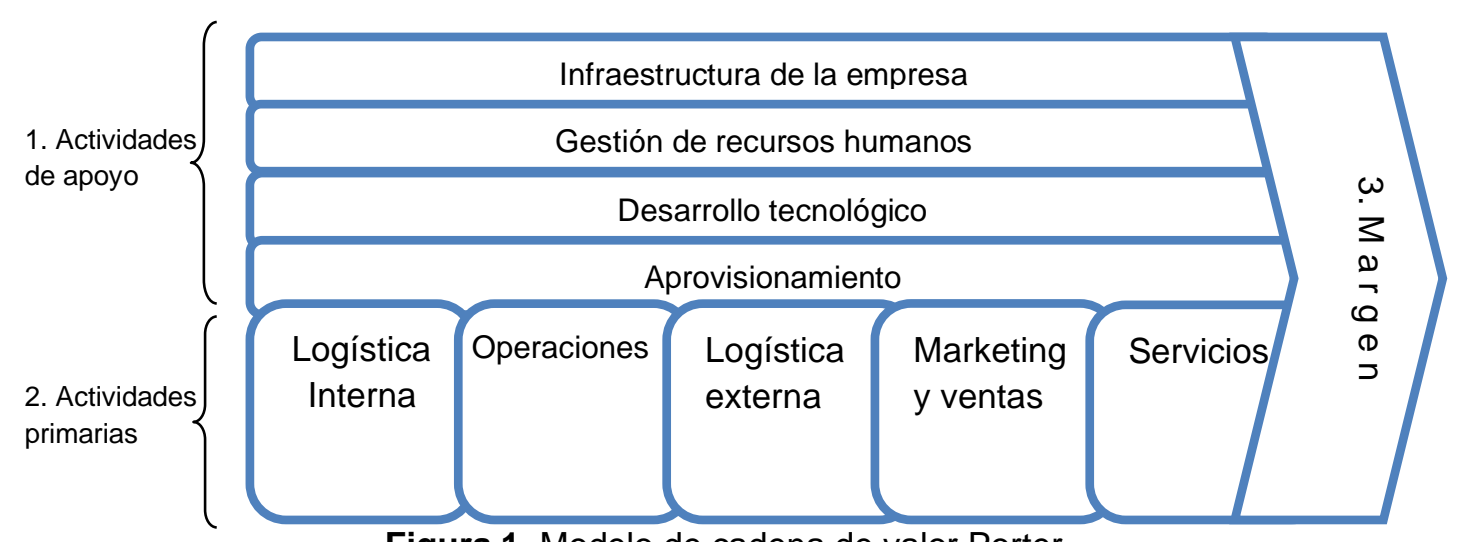

Figura 1. Modelo de cadena de valor Porter

Fuente. Porter (1985)

\section{La cadena de valor del turismo}

De acuerdo con la Organización Mundial del Turismo (OMT. 2019) la cadena de valor del turismo es una secuencia de actividades primarias y de apoyo que resultan fundamentales a nivel estratégico para el rendimiento del sector turístico. Esta primera aproximación integra los elementos que menciona Porter (1985) sobre las actividades primarias y de soporte como fuentes de ventajas competitivas generadoras de valor. Los procesos vinculados, tales como la formulación de políticas y la planificación integrada, el desarrollo de productos y su presentación al mercado, la promoción y el marketing, la distribución, la venta y las operaciones y servicios de los destinos, constituyen las principales actividades primarias de la cadena de valor del turismo.

Entre las actividades de apoyo se incluyen el transporte y la infraestructura, el desarrollo de recursos humanos, el desarrollo de tecnologías y sistemas y otros bienes y servicios complementarios que pueden no estar relacionados con la esencia del negocio turístico, pero repercuten de manera importante en el valor del turismo.

Para Rain forest, Alliance. (2005), la cadena de valor turística es muy particular y encierra retos especiales, a diferencia de la cadena de valor de los productos industriales. Ya que en el ámbito del turismo lo que se produce y se vende es un servicio. En la actualidad, las cadenas de valor fomentan cada vez más la participación de micro, pequeñas y medianas empresas para alcanzar el crecimiento integrado de la economía de un país. Gracias a este enfoque, el desarrollo y los beneficios se dan directa y equitativamente a más sectores sociales y niveles productivos.

Para ampliar el desarrollo de cadenas de valor, Gastélum-Escalante, Patiño, González, Cárdenas, Narváez, Sierra, \& Pinochet, (como se cito en Ventura 2011) afirman que comprenden todos los bienes y servicios que entran en el suministro de los productos turísticos hacia los viajeros, así como los servicios que les ayudan a planear y ejecutar sus viajes, abarcando un conglomerado de sectores independientes y relacionadas, como: hotelería, gastronomía, construcción, comercio, así como diferentes actividades expuestas mediante empresas operadoras tanto en los países que residen los viajeros, como en los países que los reciben. Gastélum-Escalante et.al. (2018) (como se cito en Ventura 2011) plantea cómo la cadena (Figura 2) empieza con los establecimientos que 
facilitan la decisión de viajar para el turista, asesorándolo, incidiendo sobre la competencia entre los destinos turísticos, por lo que los canales de comercialización y distribución globales se convierten en eslabones fundamentales para la creación y consolidación de los destinos turísticos.

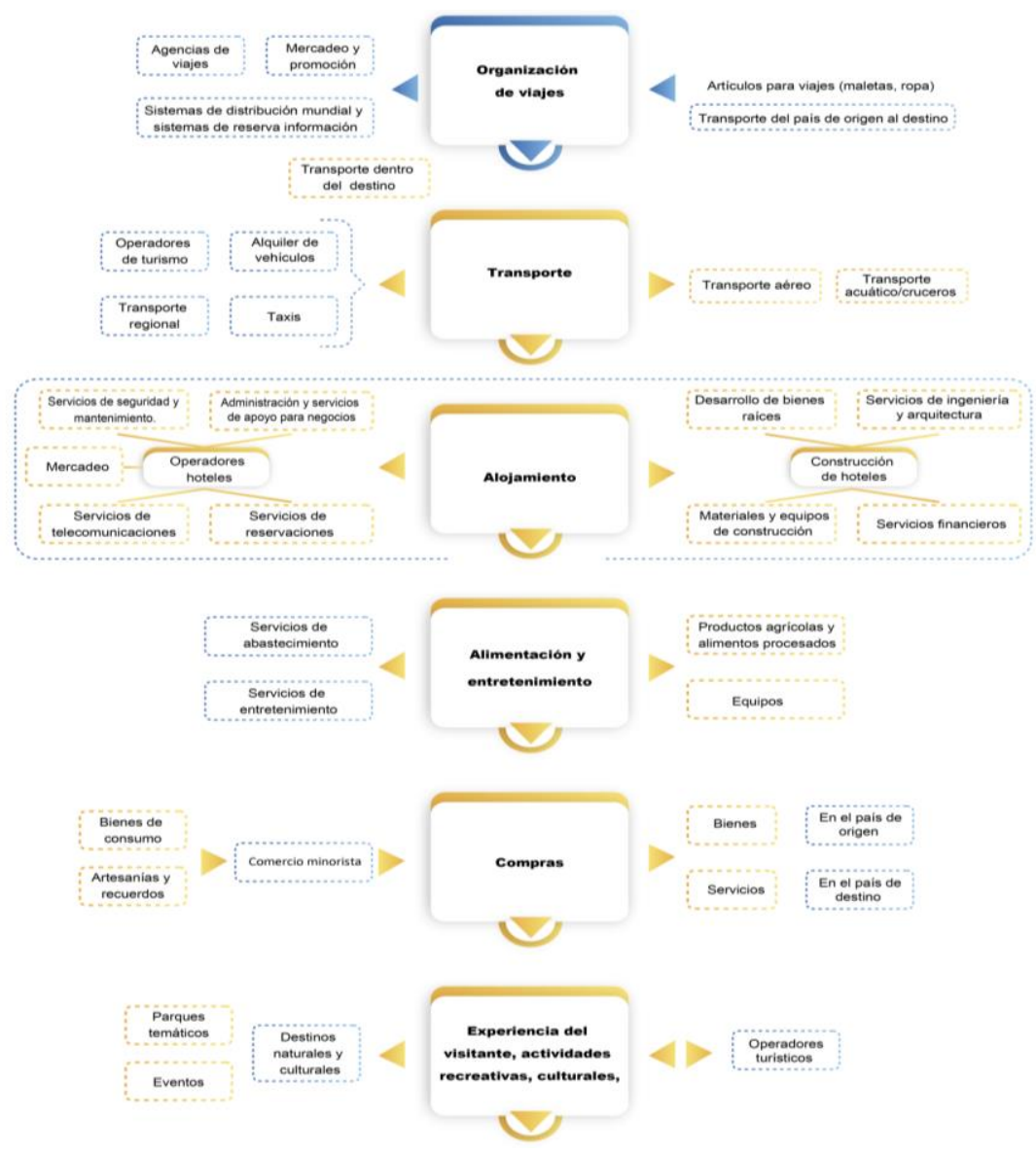

Figura 2. Cadena de valor del Turismo

Fuente. Ventura (2011)

La Figura 3 Template for creating a value chain map for tourim (2015) ilustra la complejidad de una cadena de valor turística. A la izquierda, se ilustran las actividades que tienen lugar en el país emisor y a la derecha los que tienen lugar en el país de entrada. El segmento inferior de la figura 3 representa actividades que son parte directa del sector turístico, mientras que los vinculados indirectamente al sector turístico son representados en el segmento superior. El potencial de desarrollo del turismo se maximiza en países que logran explotar los vínculos indirectos de manera óptima y la figura 3 ilustra una amplia gama de actividades pueden considerarse relevantes para lograrlo.

La posición que adopten los proveedores de los países en desarrollo en la cadena de valor del turismo puede ser otro determinante importante del potencial de crecimiento y reducción de la pobreza tiene sector turismo. Se pueden obtener considerables beneficios, por ejemplo, en el segmento de organización y reserva, las actividades relacionadas con este segmento tienen lugar en el país emisor y suelen estar en manos 
de operadores turísticos o agentes de viajes registrados en esos países (Christian, 2012). A nivel de operaciones turísticas, una considerable participación del mercado está en manos de un pequeño número de operadores (Christian y Nathan, 2013). A pesar de esta estructura de mercado concentrada, el segmento de operadores turísticos parece ser muy discutible ya que se caracteriza por un alto nivel de entrada y salida del sector (Sinclair, 1998). También es el caso de que la evolución de la información, la tecnología está cambiando el papel de los diferentes actores en la organización y el segmento de reservas, ya que facilitan que los proveedores de servicios en los países de destino lleguen directamente a clientes potenciales en el extranjero (OCDE, 2008).

Dentro del país de destino, las actividades relacionadas con el turismo incluyen actividades relacionadas con transporte regional, nacional, alojamiento y alimentos y bebidas, de acuerdo a la figura. 3 la organización de excursiones dentro del país constituye otro subcomponente de la cadena de valor del turismo; mejora las actividades turísticas en segmentos individuales de la cadena de valor puede incrementar los beneficios del turismo para un país. Por lo tanto, para mejorar el impacto de la industria del turismo en desarrollo, los países pueden considerar la creación de condiciones que faciliten a los proveedores de países conectarse a cadenas de valor, ingresar al segmento de organización de viajes y actualizar los segmentos individuales de la cadena de valor. Además, se pueden crear condiciones que estimulan los vínculos hacia atrás y hacia adelante de las actividades turísticas con otros segmentos de la economía. (DEVCO and UNWTO, 2013)

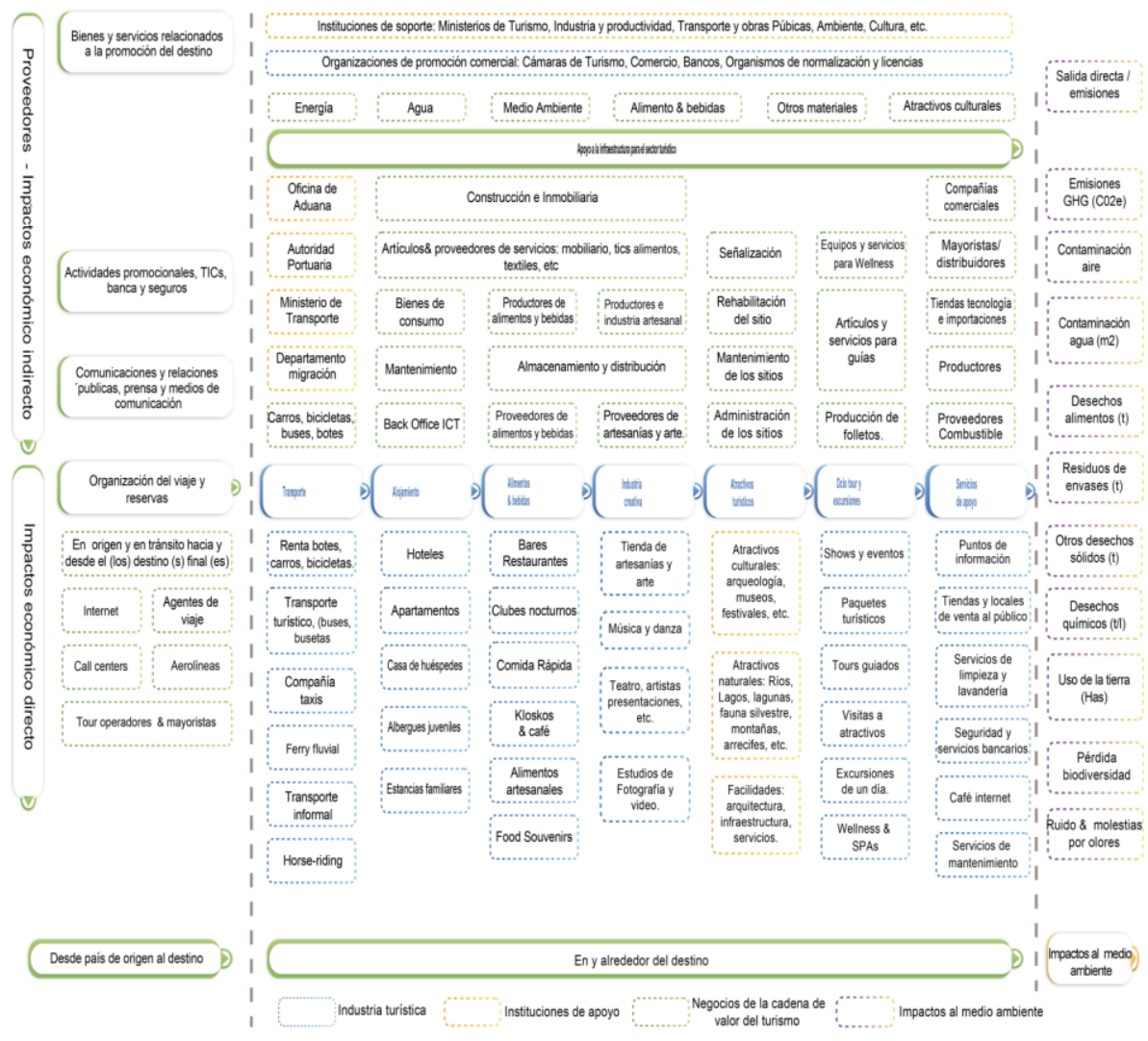

Figura 3.Template for creating value chain map for tourism Fuente. United Nations Environment programme from ITC UNWTO (2015) 


\section{Análisis de la cadena de valor del turismo en Ecuador}

La Vicepresidencia de la República del Ecuador a través de la Comisión Económica para América Latina y el Caribe CEPAL (2013) presentó los hallazgos identificados respecto a uno de los rubros más importantes de la economía ecuatoriana, el Turismo; donde se refleja los factores que definen la cadena productiva, sus fortalezas, oportunidades, debilidades, amenazas e instrumentos de política para fortalecer la cadena productiva.

Al respecto de la descripción de la cadena productiva (cadena de valor del turismo) refiere a los elementos que se ilustran en la figura 2, además se realiza un dimensionamiento de la cadena como se muestra en la tabla 1 .

Tabla 1. Ecuador. Dimensionamiento de la Cadena de Valor del Turismo (En miles de visitantes, millones de dólares y porcentaje)

\begin{tabular}{lrrr}
\hline & $\mathbf{2 0 0 6}$ & $\mathbf{2 0 1 0}$ & $\mathbf{2 0 1 3}$ \\
\hline A. Nivel de actividad & 841 & 1047 & 1366 \\
Llegada de turistas internacionales (miles de visitantes) & 733 & 899 & 1156 \\
Salida de Turistas hacia el exterior (miles de visitantes & & 9068 & \\
Turismo interno (miles de visitantes) & & & \\
B. Incidencia en la economía & 1,9 & 2 & 1,9 \\
Participación directa en el PIB(\%) & 4,8 & 5,6 & 5,3 \\
Participación total en el PIB (\%) & 4,3 & 4 & 3,8 \\
Participación en la formación bruta de capital (\%) & 3,5 & 4 & 4,5 \\
Participación en las exportaciones totales de bienes y servicios (\%) & 47,5 & 53,2 & 60,8 \\
Participación en las exportaciones de servicios (\%) & & & \\
C. Contribución a la balanza de pago & 492 & 786,6 & 1251,2 \\
Ingresos de divisas por concepto de viajes (millones de dólares) & 706,2 & 863,4 & 987,1 \\
Egresos de divisas por concepto de viajes (millones de dólares) & $-241,1$ & $-76,8$ & 264,1 \\
Saldo (millones de dólares) & & & \\
D. Perfil de los agentes & 13503 & 17778 & 21070 \\
Número de establecimientos & & & 90,9 \\
Porcentaje de micro y pequeñas empresas(\%) & & & \\
Porcentaje de empresas extranjeras (\%) & & & \\
E. Empleo & 78500 & 92800 & 114108 \\
Número de ocupados & & 60 & \\
Tasa de informalidad (\%) & & & \\
Salarios & & & \\
\hline
\end{tabular}

Fuente:Calderón 2015. Comisión Económica para América Latina y el Caribe (CEPAL), sobre la base de información del Ministerio de Turismo (MINTUR), Banco Central de Ecuador, Organización Mundial del Turismo (OMT), Superintendencia de Compañía.

\section{Turismo en áreas protegidas.}

Las áreas protegidas son el espacio de representación de la diversidad de ecosistemas, especies, paisajes y culturas. El patrimonio natural y cultural que protegen estas áreas representa un compromiso con la conservación de la biodiversidad y de la historia, y a su vez, contribuye al desarrollo de espacios donde crecientemente visitantes nacionales y extranjeros pueden descubrir, disfrutar, aprender y conectarse con la naturaleza. (Subsecretaria de Turismo. Chile. 2015) 
A nivel internacional, las visitas a áreas protegidas aumentan cada año, al igual que el gasto turístico asociado a las mismas. Según estadísticas de la Unión Internacional de Conservación de la Naturaleza (IUCN), se reciben 8 mil millones de visitantes por año. Los turistas en áreas protegidas a nivel global, gastan más de US\$ 600 mil millones en el país de destino, además de otros gastos no asociados a servicios de turismo propiamente, que corresponden a US\$ 250 mil millones. Secretariat of the Convention on Biological Diversity, (2015).

En el Ecuador, el Sistema Nacional de Áreas Protegidas y otras áreas de conservación establecen a la biodiversidad como un sector estratégico y de interés público (Constitución 2008, Arts. 313, 400). Esto se fortalece al considerar al patrimonio natural del Ecuador como único e invaluable y manifestar que su gestión se llevará a cabo de acuerdo al ordenamiento territorial y zonificación ecológica (Constitución 2008, Art. 404). Las áreas protegidas y áreas de conservación en el país están ubicadas en zonas rurales principalmente, por lo que su gestión propende al mantenimiento y conservación de la biodiversidad, pero también busca mejorar las condiciones de vida de las poblaciones que los acogen, a través del turismo sostenible. (MINTUR.2019)

CEPAL (2015), definió al SNAP del Ecuador como el mayor potencial atractivo turístico dentro de la matriz productiva. En efecto, contribuye con el 32\% (USD 446 millones) de los ingresos turísticos nacionales registrados en el año 2013, este dato es obtenido del Patrimonio de Áreas Naturales del Estado (PANE) y Parque Nacional Galápagos (PNG) Igualmente, el MAE (2015) establece que el 68\% de los turistas extranjeros manifiestan su principal motivación para viajar al Ecuador es visitar parques naturales.

En la siguiente tabla se muestra el ingreso total de turistas (nacionales y extranjeros) evidenciando un crecimiento exponencial de visitantes nacionales en áreas protegidas a partir del año 2012 según los datos disponibles en 27 áreas, llegando a un número total de 796.044 visitantes. Este crecimiento se debe a que a partir del año 2012 se establece la gratuidad para el ingreso al Patrimonio de Áreas Naturales del Estado (PANE), excepto Parque Nacional Galápagos.

Tabla 2. Número de visitantes al Patrimonio de Áreas Naturales del Estado (PANE)

\begin{tabular}{lclll}
\hline Año & Nro. APS & Nacionales & Extranjeros & Total \\
\hline $\mathbf{2 0 1 0}$ & 21 & 275.518 & 120.420 & 395.938 \\
$\mathbf{2 0 1 1}$ & 23 & 441.176 & 168.417 & 609.593 \\
$\mathbf{2 0 1 2}$ & 27 & 796.044 & 201.104 & 997.148 \\
$\mathbf{2 0 1 3}$ & 29 & 979.404 & 263.659 & 1.243 .063 \\
$\mathbf{2 0 1 4}$ & 33 & 1.461 .519 & 469.437 & 1.930 .956 \\
$\mathbf{2 0 1 5}$ & 32 & 1.584 .687 & 389.522 & 1.974 .209 \\
$\mathbf{2 0 1 6}$ & 32 & 1.557 .339 & 284.994 & 1.842 .333 \\
$\mathbf{2 0 1 7}$ & 31 & 1.582 .029 & 318.061 & 1.900 .090 \\
$\mathbf{2 0 1 8}$ & 31 & 1.490 .882 & 342.989 & 1.833 .871 \\
\hline \multicolumn{5}{l}{}
\end{tabular}

Fuente: Ministerio del Ambiente 2018

Respecto a la visitación de turistas extranjeros excepto Parque Nacional Galápagos, existen datos mensuales y estables en 19 áreas protegidas (de 33 con registros) desde el año 2010 hasta el año 2018. Algunas áreas como el Parque Nacional Yasuní, 
Podocarpus, Cayambe-Coca, Cajas, Reserva Ecológica El Ángel, Producción de Fauna Cuyabeno registran incrementos estables. (MINTUR.2019).

\section{La actividad turística en el Parque Nacional Yasuní.}

El Parque Nacional Yasuní (PNY) se encuentra en el centro oriente de la Amazonía ecuatoriana, es el área protegida más grande y biodiversa del Ecuador continental tiene una extensión de más de un millón de hectáreas, en la actualidad, las operaciones turísticas se concentran, en las zonas de las riveras de los ríos Napo y Yasuní, por (MAE. 2016).

El desarrollo turístico en la zona de amortiguamiento y parte del Parque Nacional Yasuní inicia hace 35 años, donde se instalan seis lodges, Sacha y La Selva fueron los primeros, así como la operación del Flotel Orellana, que ofrecía tours de selva por diferentes comunidades y sitios de interés específicos en las riveras de los ríos, por (MAE. 2016)

La actividad turística en el PNY se ha incrementado de manera considerable, así lo demuestra la información del año 2000 con 130 visitantes, evolucionando a 3000 en el año 2010, un máximo de 13.876 en el registro del año 2014, finalmente 11.213 visitantes en el año 2019, por (MAE. 2019).

De acuerdo a la zonificación de los sitios de visita, según escenarios en el Parque Nacional Yasuní se ha identificado seis sitios de visita: Indillama, Nueva Providencia, Añangu, Tambococha, Mandarapinga y Shiripuno. En estos sitios realizan operación turística 20 operadores, 12 privados y 8 comunitarios.

\section{Metodología.}

El Parque Nacional Yasuní ubicado en el sector centro oriental de la región amazónica ha sido reconocido como el lugar de mayor diversidad del hemisferio occidental (Bass, Finer et al, 2010). Sus ecosistemas únicos han evolucionado desde la formación de la selva Amazónica hace 25 millones de años, han sobrevivido los rigores de las glaciaciones del Pleistoceno (Hoorn, 2006) y poseen una excepcional resilencia frente al cambio climático futuro.

Se encuentra entre los 200-400 m.s.n.m., con una extensión de más de un millón de hectáreas, el Yasuní fue declarado Parque Nacional en 1979 y diez años más tarde la UNESCO lo estableció como Reserva de Biosfera, por su alta biodiversidad respecto al nivel de especies, de ecosistemas y genes, además el albergue de grupos indígenas no contactados que mantienen aislamiento voluntario, por lo cual una parte del Parque fue delimitada como Zona Intangible en el año 2006.

De acuerdo al Plan de Manejo del área protegida más de 50.000 hectáreas corresponden a las de uso, con fines turísticos, por (MAE 2016) 


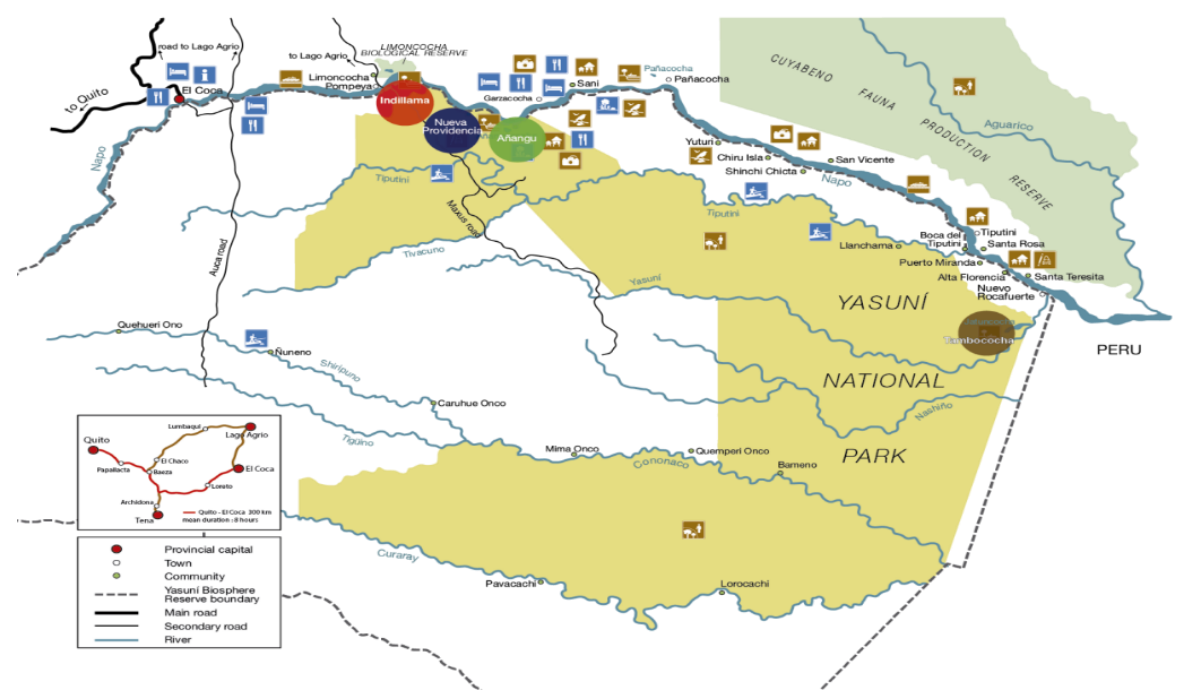

Figura 4. Mapa de atractivos turísticos del Parque Nacional Yasuní Fuente: Ministerio del Ambiente 2019

La investigación se fundamenta en el enfoque mixto que hace referencia al uso de datos numéricos así como de las características y experiencias de quienes se involucran directamente con la operación turística del Parque Nacional Yasuní, hechos que son necesarios identificar y conocer in situ a través de la investigación exploratoria y descriptiva. (Vara, Horna. 2015).

En este contexto, se realizó visitas a los operadores turísticos privados y comunitarios, con la finalidad de recopilar información a través de la aplicación de entrevistas estructuradas, cuyas variables fueron procesadas mediante análisis estadístico multivariado, y que permitió realizar la descripción comparativa y de correlación de los datos de la investigación.

La investigación exploratoria documental permitió identificar las variables socio demográficas que reflejan el perfil del visitante del Parque Nacional Yasuní. Con el procesamiento estadístico se identificaron los componentes que agrupan a los elementos de la cadena de valor del área protegida.

Finalmente, la investigación descriptiva permitió diseñar un mapa que refleja la cadena de valor de turismo Parque Nacional Yasuní, con elementos que son parte de las actividades primarias generadoras de valor, a partir de los componentes establecidos en el análisis multivariado.

\section{Resultados.}

El Plan de Manejo de visitantes del PNY (2016), describe los resultados para determinar el perfil de visitante, con información que evidencia información demográfica y preferencias figura 5.El mismo plan refleja la variable de satisfacción del visitante a partir de la percepción que tiene los mismos sobre los servicios que se ofertan en los diferentes sitios de visita, el resultado cuantitativo de entre cuatro variables establecidas indica Muy bueno. 


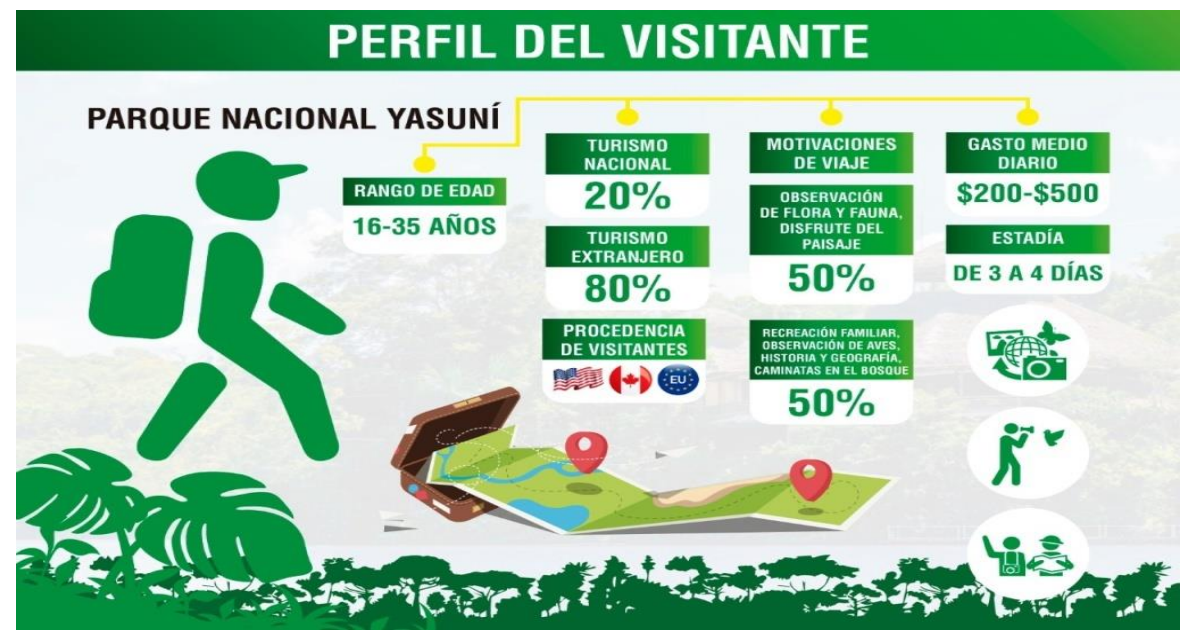

Figura 5. Infografía creada a partir del Perfil del visitante PNY Fuente. Jefatura Parque Nacional Yasuní

Para el estudio se tomó como referentes a los operadores turísticos que cuentan con registro en el Catastro Nacional de Turismo y Patente de Operación Ambiental de actividades turísticas emitida por la Autoridad Ambiental. (14 de 21 operadores identificados).

Tabla 3.Empresas y tour operadores legalmente registrados

\begin{tabular}{|c|c|c|c|c|c|}
\hline No & Operador & Ubicación & $\begin{array}{l}\text { Área } \\
\text { Protegida }\end{array}$ & Registro turístico & Patente ambiental \\
\hline 1 & CTC Napo Wild Life Center & $\begin{array}{l}\text { Comunidad } \\
\text { KichwaAñangu }\end{array}$ & PNY & 2201510218 & PAOT204991000956 \\
\hline 2 & CTC Napo Cultural Center & $\begin{array}{l}\text { Comunidad } \\
\text { KichwaAñangu }\end{array}$ & PNY & 2201510399 & РАОТ204991000956 \\
\hline 3 & CTC Sani Isla & $\begin{array}{l}\text { Comunidad } \\
\text { KichwaSani Isla }\end{array}$ & $\mathrm{ZA}$ & 2104510078 & \\
\hline 4 & Sacha Lodge & $\begin{array}{l}\text { Comunidad Kichwa } \\
\text { Pilche }\end{array}$ & $Z A-P N Y$ & KSHUAJ00002134 & \\
\hline 5 & Selva Amazon Ecolodge\& Spa & $\begin{array}{l}\text { Comunidad Kichwa } \\
\text { Pilche }\end{array}$ & $Z A-P N Y$ & KSHUAJ00002136 & РАОT205270001027 \\
\hline 6 & AdvangeTravel & $\begin{array}{l}\text { Ninguna (Manatee- } \\
\text { Anacaonda) }\end{array}$ & ZA - PNY & PUIOOI-00000766 & РАОТ204783000875 \\
\hline 7 & Witoto Tours & $\begin{array}{l}\text { Francisco de } \\
\text { Orellanag }\end{array}$ & ZA - PNY & QORLOI00001032 & \\
\hline 8 & SumakAllpa Tour Cia Ltda. & $\begin{array}{l}\text { Francisco de } \\
\text { Orellana }\end{array}$ & $Z A-P N Y$ & QORLOI00000534 & РАОТ314793000834 \\
\hline 9 & ShiripunoLodge\&Research & $\begin{array}{l}\text { Comunidad } \\
\text { WaoraniNoeneno }\end{array}$ & ZITT - ZA & 2201500297 & \\
\hline 10 & Amazon Travel Tour & $\begin{array}{l}\text { Francisco de } \\
\text { Orellana }\end{array}$ & $Z A-P N Y$ & QORLOI00000243 & РАОТ316080000950 \\
\hline 11 & $\begin{array}{l}\text { Mandari Panga Yasuni Jungle } \\
\text { Exp }\end{array}$ & $\begin{array}{l}\text { Comunidad Kichwa } \\
\text { Mandaripanga }\end{array}$ & PNY & QORLAJ00003549 & РАOT312089000965 \\
\hline 12 & YuturiCía Ltda. & $\begin{array}{l}\text { YarinaLodge, } \\
\text { comunidad Kichwa } \\
\text { Pañacoha }\end{array}$ & $\mathrm{ZA}$ & PUIOOI-00000817 & \\
\hline 13 & Amazon Wildlife / C. Indilllama & $\begin{array}{l}\text { Comunidad } \\
\text { Kichwalndillama }\end{array}$ & PNY & QORLOI00000963 & \\
\hline 14 & CTC Sach & $\begin{array}{l}\text { Comunidad Alta } \\
\text { Florencia }\end{array}$ & ZA- PNY & 2202500274 & RNAB31168950442 \\
\hline
\end{tabular}

Fuente: Datos Obtenidos Catastro Nacional MINTUR 2020. Jefatura Parque Nacional Yasuní. MAE.2020. Parque Nacional Yasuní (PNY)- Zona amortiguamieno (ZA) 
A partir de la información identificada y procesada, la investigación recoge una base de datos conformada por 14 sujetos de estudio y 34 variables, el instrumento de recopilación fue validado por Alpha de Cronbach a través del procesador estadístico IBM SPSS Statics 22. El resultado determinó un alto grado de fiabilidad según el análisis de todos los ítems propuestos.

En el análisis estadístico con 14 operadores turísticos y 34 variables, 2 variables no presentaron variabilidad porque su representación fue 0 . A continuación se detalla las variables definidas para poder realizar análisis mutivariado con la técnica componentes principales.

Tabla 4. Variables del sistema y su desviación estándar

\begin{tabular}{|c|c|c|}
\hline Variables & $\mathbf{N}$ & $\begin{array}{l}\text { Desviación } \\
\text { estándar }\end{array}$ \\
\hline Años de Operación & 14 & 1,0509 \\
\hline Con qué número de agentes de viaje trabaja & 14 & 1,21574 \\
\hline Con cuántas líneas aéreas nacionales trabaja & 14 & 0,63332 \\
\hline Cuántas CIAS Transporte turístico terrestre utiliza & 14 & 0,66299 \\
\hline Cuántas CIAS transporte público (buses) utiliza & 14 & 0,75955 \\
\hline Cuántas CIAS de busetas, camionetas o taxis utiliza & 14 & 0 \\
\hline Con cuántas unidades de transporte fluvial cuenta & 14 & 0,94926 \\
\hline Realiza renta de triciclos & 14 & 0,36314 \\
\hline Número de atractivos turísticos naturales visita & 14 & 0,51887 \\
\hline Número de atractivos turísticos culturales visita & 14 & 1,20667 \\
\hline Con cuántos guías nacionales cuenta & 14 & 0,94926 \\
\hline Con cuántos guías locales trabaja & 14 & 0,63332 \\
\hline Utiliza guías intérpretes & 14 & 0,51887 \\
\hline Qué tipo de alojamiento utilizan en Coca & 14 & 0,92878 \\
\hline Qué tipo de alojamiento utiliza durante su operación & 14 & 2,11873 \\
\hline Utiliza restaurantes y/o cafeterías & 14 & 1,12171 \\
\hline Utiliza comedores de comida típica & 14 & 0,51355 \\
\hline Visita sitios donde expenden alimentos artesanales & 14 & 0,51887 \\
\hline Visita sitios donde expenden FoodSouvenirs & 14 & 0,49725 \\
\hline Visita tiendas de artesanías & 14 & 0,49725 \\
\hline Atractivos de la ciudad de El Coca & 14 & 0,75593 \\
\hline Número de comunidades que se benefician de su operación turística & 14 & 0,53452 \\
\hline Número de paquetes turísticos o programas establecidos & 14 & 0,87706 \\
\hline Realiza viajes cortos de un día & 14 & 0,36314 \\
\hline Tiene algún servicio relacionado a Wellness and SPA & 14 & 0,26726 \\
\hline Utiliza Centro de Información Turística & 14 & 0,36314 \\
\hline Utiliza tienda de abarrotes & 14 & 0,49725 \\
\hline Utiliza servicio de lavandería & 14 & 0,42582 \\
\hline Utiliza servicios bancarios y de seguridad & 14 & 0 \\
\hline Utiliza servicios de mantenimiento & 14 & 0,51887 \\
\hline Utiliza o renta VIP Lounge & 14 & 0,42582 \\
\hline Gasto promedio diario en su operación & 14 & 1,38477 \\
\hline Priorice los principales impactos & 14 & 1,28388 \\
\hline Acciones de mejora que influyan en competitividad turística & 14 & 2,11873 \\
\hline $\mathrm{N}$ válido (por lista) & 14 & \\
\hline
\end{tabular}

Fuente: Datos obtenidos con el procesador estadístico IBM SPSS Statistics 22. Algoritmos Chicago: IBM SPSS Inc. 
Se realizó un análisis descriptivo al encontrar la desviación estándar y de esta manera determinar las variables que tienen desviación igual a cero y omitir su uso para el cálculo de los componentes principales.

Para realizar el análisis de componentes en primer lugar se realizó el análisis de suficiencia general Kaiser - Meyer - Olklin $(\mathrm{KMO}=0,72)$; al ser superior a 0,5 el KMO es procedente realizar el análisis de Componentes Principales.

De los resultados obtenidos en el tratamiento estadístico se muestra en la tabla 5., se puede decir que con el $63.89 \%$ de varianza total se puede explicar el sistema con cuatro componentes nuevas variables.

Tabla 5.Varianza total explicada

\begin{tabular}{|c|c|c|c|c|c|c|}
\hline \multirow[t]{2}{*}{ Variable } & \multicolumn{3}{|c|}{ Autovalores iniciales } & \multicolumn{3}{|c|}{$\begin{array}{c}\text { Sumas de extracción de cargas al } \\
\text { cuadrado }\end{array}$} \\
\hline & Total & $\%$ de varianza & $\begin{array}{c}\% \\
\text { acumulado }\end{array}$ & Total & $\begin{array}{c}\% \text { de } \\
\text { varianza }\end{array}$ & $\begin{array}{c}\% \\
\text { acumulado }\end{array}$ \\
\hline 1 & 7,678 & 23,995 & 23,995 & 7,678 & 23,995 & 23,995 \\
\hline 2 & 5,978 & 18,681 & 42,676 & 5,978 & 18,681 & 42,676 \\
\hline 3 & 3,547 & 11,086 & 53,761 & 3,547 & 11,086 & 53,761 \\
\hline 4 & 3,243 & 10,135 & 63,896 & 3,243 & 10,135 & 63,896 \\
\hline 5 & 2,761 & 8,63 & 72,526 & 2,761 & 8,63 & 72,526 \\
\hline 6 & 2,281 & 7,127 & 79,652 & 2,281 & 7,127 & 79,652 \\
\hline 7 & 2,051 & 6,408 & 86,06 & 2,051 & 6,408 & 86,06 \\
\hline 8 & 1,864 & 5,825 & 91,885 & 1,864 & 5,825 & 91,885 \\
\hline 9 & 1,249 & 3,902 & 95,787 & 1,249 & 3,902 & 95,787 \\
\hline 10 & 0,78 & 2,437 & 98,224 & & & \\
\hline 11 & 0,499 & 1,558 & 99,782 & & & \\
\hline 12 & 0,052 & 0,163 & 99,945 & & & \\
\hline 13 & 0,018 & 0,055 & 100 & & & \\
\hline 14 & $7,86 \mathrm{E}-16$ & $2,46 \mathrm{E}-15$ & 100 & & & \\
\hline 15 & $5,72 \mathrm{E}-16$ & $1,79 \mathrm{E}-15$ & 100 & & & \\
\hline 16 & 4,38E-16 & $1,37 \mathrm{E}-15$ & 100 & & & \\
\hline 17 & $3,39 \mathrm{E}-16$ & $1,06 \mathrm{E}-15$ & 100 & & & \\
\hline 18 & $3,04 \mathrm{E}-16$ & $9,48 \mathrm{E}-16$ & 100 & & & \\
\hline 19 & $2,81 \mathrm{E}-16$ & $8,78 \mathrm{E}-16$ & 100 & & & \\
\hline 20 & $2,00 \mathrm{E}-16$ & $6,26 \mathrm{E}-16$ & 100 & & & \\
\hline 21 & $1,36 \mathrm{E}-16$ & $4,26 \mathrm{E}-16$ & 100 & & & \\
\hline 22 & $2,85 \mathrm{E}-17$ & $8,90 \mathrm{E}-17$ & 100 & & & \\
\hline 23 & $5,58 \mathrm{E}-18$ & $1,74 \mathrm{E}-17$ & 100 & & & \\
\hline 24 & $-5,52 \mathrm{E}-17$ & $-1,73 \mathrm{E}-16$ & 100 & & & \\
\hline 25 & $-1,05 \mathrm{E}-16$ & $-3,30 \mathrm{E}-16$ & 100 & & & \\
\hline 26 & $-2,25 \mathrm{E}-16$ & $-7,04 \mathrm{E}-16$ & 100 & & & \\
\hline 27 & $-2,52 \mathrm{E}-16$ & $-7,88 \mathrm{E}-16$ & 100 & & & \\
\hline 28 & $-4,28 \mathrm{E}-16$ & $-1,34 \mathrm{E}-15$ & 100 & & & \\
\hline 29 & $-5,17 \mathrm{E}-16$ & $-1,61 \mathrm{E}-15$ & 100 & & & \\
\hline 30 & $-6,81 \mathrm{E}-16$ & $-2,13 \mathrm{E}-15$ & 100 & & & \\
\hline 31 & $-8,47 \mathrm{E}-16$ & $-2,65 \mathrm{E}-15$ & 100 & & & \\
\hline 32 & $-1,09 \mathrm{E}-15$ & $-3,40 \mathrm{E}-15$ & 100 & & & \\
\hline
\end{tabular}

Fuente. Datos obtenidos con el procesador estadístico IBM SPSSStatistics 22. Algoritmos Chicago: IBM SPSSInc.Método de extracción: análisis de componentes principales.

A continuación la gráfica de sedimentación evidencia los componentes del sistema estadístico en análisis, a partir del componente 5 el nivel de significancia empieza a 
disminuir, en esta relación se valida lo planteado en la tabla 5 respecto a la determinación de los 4 componentes principales.

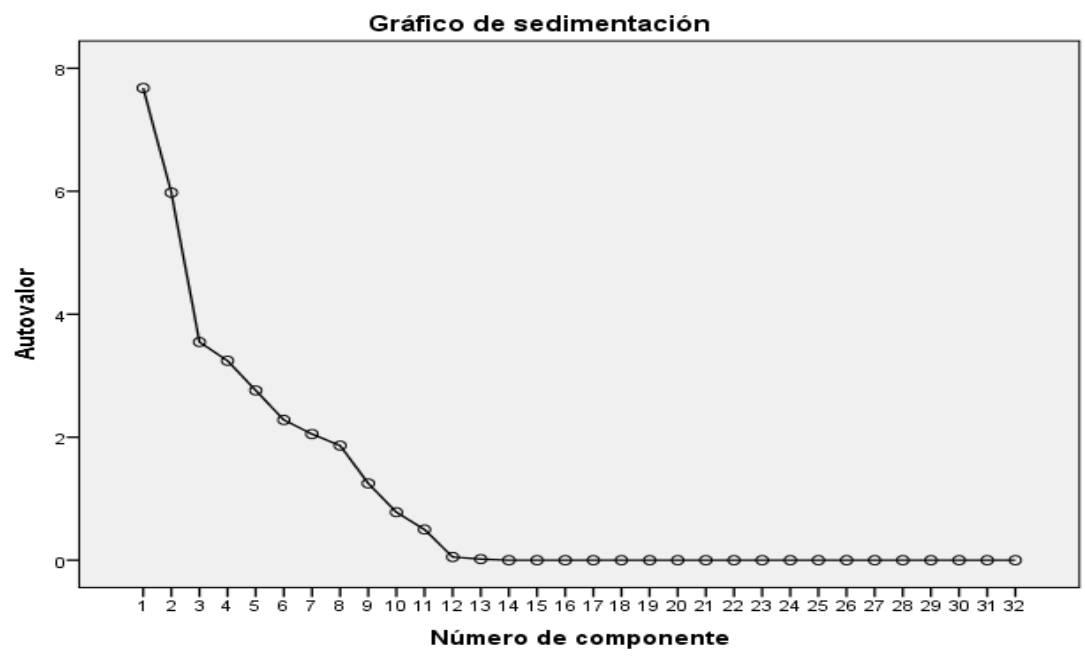

Figura 5. Gráfica de sedimentación del sistema estadístico

Según (Hair, Black, Babin, \& Anderson, 2016), las variables que tuvieran valores de peso superiores a 0.5 , es suficiente para identificar variables en cada componente seleccionado.

Tabla. 6 Matriz de componentes

\begin{tabular}{|c|c|c|c|c|c|c|c|c|c|}
\hline & \multicolumn{9}{|c|}{ Matriz de componente ${ }^{\mathrm{a}}$} \\
\hline & \multicolumn{9}{|c|}{ Componentes } \\
\hline & 1 & 2 & 3 & 4 & 5 & 6 & 7 & 8 & 9 \\
\hline \multirow[t]{2}{*}{ Años de Operación } & & - & - & & & - & & & \\
\hline & 0,759 & 0,039 & 0,482 & 0,117 & 0,212 & 0,021 & 0,068 & 0,151 & 0,304 \\
\hline \multirow{2}{*}{$\begin{array}{l}\text { Con qué número de operadoras turísticas } \\
\text { trabaja }\end{array}$} & & & & & 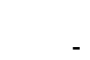 & & & - & \\
\hline & 0,475 & 0,667 & 0,477 & 0,07 & 0,022 & 0,098 & 0,076 & 0,088 & 0,127 \\
\hline \multirow{2}{*}{$\begin{array}{l}\text { Con cuántas líneas aéreas nacionales } \\
\text { trabaja }\end{array}$} & & & - & & & - & - & - & \\
\hline & 0,175 & 0,642 & 0,346 & 0,324 & 0,366 & 0,084 & 0,149 & 0,376 & 0,032 \\
\hline $\begin{array}{l}\text { Cuántas CIAS Transporte turístico } \\
\text { terrestre utiliza }\end{array}$ & & & & & & & & & \\
\hline \multirow{2}{*}{$\begin{array}{l}\text { Cuántas CIAS transporte público (buses) } \\
\text { utiliza }\end{array}$} & 0,103 & $\begin{array}{r}0,04 \\
\text { - }\end{array}$ & $\begin{array}{r}0,246 \\
\text { - }\end{array}$ & 0,495 & 0,486 & 0,136 & 0,116 & 0,243 & $-0,31$ \\
\hline & 0,498 & 0,615 & 0,344 & 0,291 & 0,314 & 0,156 & 0,193 & 0,062 & 0,005 \\
\hline $\begin{array}{l}\text { Con cuántas unidades de transporte } \\
\text { fluvial cuenta }\end{array}$ & 0844 & 0214 & 0415 & 0109 & 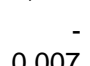 & 78 & 16 & ?ח & ? \\
\hline \multicolumn{10}{|l|}{ Realizarenta de triciclos } \\
\hline \multirow{3}{*}{$\begin{array}{l}\text { Número de atractivos turísticos naturales } \\
\text { visita } \\
\text { Número de atractivos turísticos culturales } \\
\text { visita }\end{array}$} & 0,191 & 0,278 & 0,695 & 0,399 & 0,331 & 0,072 & 0,002 & 0,273 & 0,031 \\
\hline & 0,497 & 0,539 & 0,023 & 0,473 & $0,128^{-}$ & $0,094^{-}$ & 0,191 & $0,086^{-}$ & 0,309 \\
\hline & & & & - & 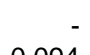 & & & - & \\
\hline \multirow[t]{2}{*}{ Con cuántos guías nacionales cuenta } & 0,052 & 0,673 & 0,219 & $\begin{array}{r}0,227 \\
-\end{array}$ & $\begin{array}{r}0,094 \\
-\end{array}$ & 0,294 & $\begin{array}{r}0,279 \\
\text { - }\end{array}$ & 0,486 & 0,144 \\
\hline & 0,259 & $-0,68$ & 0,349 & 0,079 & 0,234 & 0,222 & 0,153 & 0,146 & 0,373 \\
\hline \multirow{2}{*}{ Con cuántos guías locales trabaja } & & & & & & & & & \\
\hline & 0,54 & 0,383 & 0,439 & 0,104 & $-0,4$ & 0,112 & 0,088 & 0,243 & 0,307 \\
\hline Utilizaguíasintérpretes & 0,886 & 0,38 & 0,049 & 0,152 & 0,048 & 0,019 & 0,007 & 0,171 & 0,04 \\
\hline \multirow{3}{*}{$\begin{array}{l}\text { Qué tipo de alojamiento utilizan en Coca } \\
\text { Qué tipo de alojamiento utiliza durante su } \\
\text { operación }\end{array}$} & & & & $\begin{array}{lll}- & \end{array}$ & & - & - & & \\
\hline & 0,421 & 0,089 & 0,345 & 0,069 & 0,776 & 0,139 & 0,145 & 0,029 & 0,012 \\
\hline & 0,418 & 0,28 & 0,453 & 0,385 & 0,385 & 0,286 & 0,335 & 0,1 & 0,108 \\
\hline
\end{tabular}




\begin{tabular}{|c|c|c|c|c|c|c|c|c|c|}
\hline Utiliza restaurantes y/o cafeterías & & - & - & - & & & - & - & \\
\hline Utiliza comedores de comida típica & 0,285 & 0,212 & 0,436 & 0,746 & 0,213 & 0,026 & 0,039 & 0,077 & 0,173 \\
\hline Visita sitios donde alimentos artesanales & 0,644 & 0,097 & 0,022 & 0,362 & 0,35 & 0,2 & 0,192 & 0,424 & 0,203 \\
\hline $\begin{array}{l}\text { Visita sitios dondeexpenden Food } \\
\text { Souvenirs }\end{array}$ & 0,8 & 0,122 & 0,024 & 0,148 & 0,295 & 0,145 & 0,042 & 0,32 & 0,289 \\
\hline Visitatiendas de artesanías & 0,035 & 0,612 & $\begin{array}{r}0,642 \\
-\end{array}$ & $\begin{array}{r}0,229 \\
-\end{array}$ & $\begin{array}{r}0,025 \\
-\end{array}$ & 0,146 & 0,204 & $\begin{array}{r}0,301 \\
-\end{array}$ & 0,041 \\
\hline Atractivos de la ciudad del Coca & $\begin{array}{r}0,544 \\
-\end{array}$ & 0,329 & 0,164 & 0,108 & 0,105 & 0,579 & $\begin{array}{r}0,314 \\
-\end{array}$ & 0,251 & 0,165 \\
\hline $\begin{array}{l}\text { Número de comunidades que se } \\
\text { benefician de su operación turística }\end{array}$ & 0,216 & 0,106 & 0,057 & 0,555 & 0,443 & 0,285 & 0,029 & 0,342 & 0,443 \\
\hline $\begin{array}{l}\text { Número de paquetes turísticos o } \\
\text { programas tiene establecido }\end{array}$ & 0,302 & 0,017 & 0,13 & 0,075 & 0,06 & 0,375 & 0,856 & 0,041 & 0,023 \\
\hline Realiza viajes cortos de un día & 0,163 & 0,385 & 0,196 & 0,497 & 0,507 & 0,192 & 0,26 & 0,07 & 0,349 \\
\hline $\begin{array}{l}\text { Tiene algún servicio relacionado a } \\
\text { Wellness and SPA }\end{array}$ & 0,602 & 0,578 & 0,18 & 0,336 & 0,185 & 0,003 & 0,158 & 0,301 & 0,016 \\
\hline Utiliza Centro de Información Turística & 0,317 & 0,051 & 0,089 & 0,408 & 0,099 & 0,665 & 0,451 & 0,194 & 0,172 \\
\hline Utilizatienda de abarrotes & 0,602 & 0,578 & 0,18 & 0,336 & 0,185 & 0,003 & 0,158 & 0,301 & 0,016 \\
\hline Utilizaservicio de lavandería & 0,78 & 0,208 & 0,277 & 0,019 & 0,017 & $\begin{array}{r}0,099 \\
-\end{array}$ & $\begin{array}{r}0,416 \\
-\end{array}$ & 0,2 & 0,086 \\
\hline Utilizaservicios de mantenimiento & 0,426 & 0,56 & 0,66 & 0,017 & 0 & 0,008 & $\begin{array}{r}0,086 \\
-\end{array}$ & 0,234 & 0,008 \\
\hline Utiliza o renta VIP Lounge & 0,209 & 0,636 & 0,217 & 0,189 & 0,082 & 0,577 & 0,135 & 0,29 & 0,096 \\
\hline Gasto promedio diario en su operación & 0,645 & 0,085 & 0,131 & 0,277 & 0,002 & 0,559 & 0,149 & 0,167 & 0,288 \\
\hline Priorice los principalesimpactos & 0,286 & $-0,58$ & 0,139 & 0,474 & $\begin{array}{r}0,268 \\
-\end{array}$ & 0,056 & $\begin{array}{r}0,354 \\
-\end{array}$ & $-0,15$ & $-0,05$ \\
\hline $\begin{array}{l}\text { Acciones de mejora que influyan en } \\
\text { competitividad turística }\end{array}$ & 0,056 & 0,452 & 0,042 & 0,225 & 0,511 & $-0,32$ & 0,019 & 0,406 & 0,067 \\
\hline & 0,578 & 0,584 & 0,292 & 0,187 & 0,037 & 0,266 & 0,327 & 0,05 & 0,015 \\
\hline
\end{tabular}

Fuente.Datos obtenidos con el procesador estadístico IBM SPSSStatistics 22. Algoritmos Chicago: IBM SPSS Inc. Método de extracción: análisis de componentes principales.

Como se evidencia las cuatro componentes principales estarán constituidas por:

COMPONENTE 1: Años de operación, transporte público, transporte fluvial, guías locales, guías intérpretes, comedores de comida típica, alimentos artesanales, tiendas de artesanías, comunidades beneficiadas, Viajes cortos, centro de información, tienda de abarrotes, VIP Lounge, acciones para mejora de la competitividad turística.

COMPONENTE 2: Agentes de viaje, líneas aéreas nacionales, atractivos turísticos naturales, atractivos turísticos culturales, guías nacionales, alojamiento, servicios de mantenimiento, gasto promedio, impacto al ambiente. 
COMPONENTE 3: Renta de triciclos, tipo de alojamiento, food souvenirs, servicio de lavandería.

COMPONENTE 4: Compañías transporte turístico, restaurantes y cafeterías, atractivos de la ciudad de El Coca, Paquetes turísticos establecidos, servicio wellness.

Una vez obtenidas los cuatro componentes; se hace uso de las mismas para mapear la cadena de valor para el Parque Nacional Yasuní, se utiliza la metodología propuesta por United Nations Enviroment Programm UN Enviroment desde la ITC UNWTO (2015) definiendo el alcance y objetivo, así como los componentes establecidos por el análisis estadístico.

El objetivo y alcance de la cadena se encuentra sobre los elementos relacionados a los impactos económicos directos (actividades primarias Modelo Porter 1985) y no la cadena de suministros (actividades de apoyo).

La construcción de la figura 6 es el resultado del mapeo de la cadena de valor, su sistema de valor así como los impactos medioambientales considerando que la investigación refiere a un área protegida.

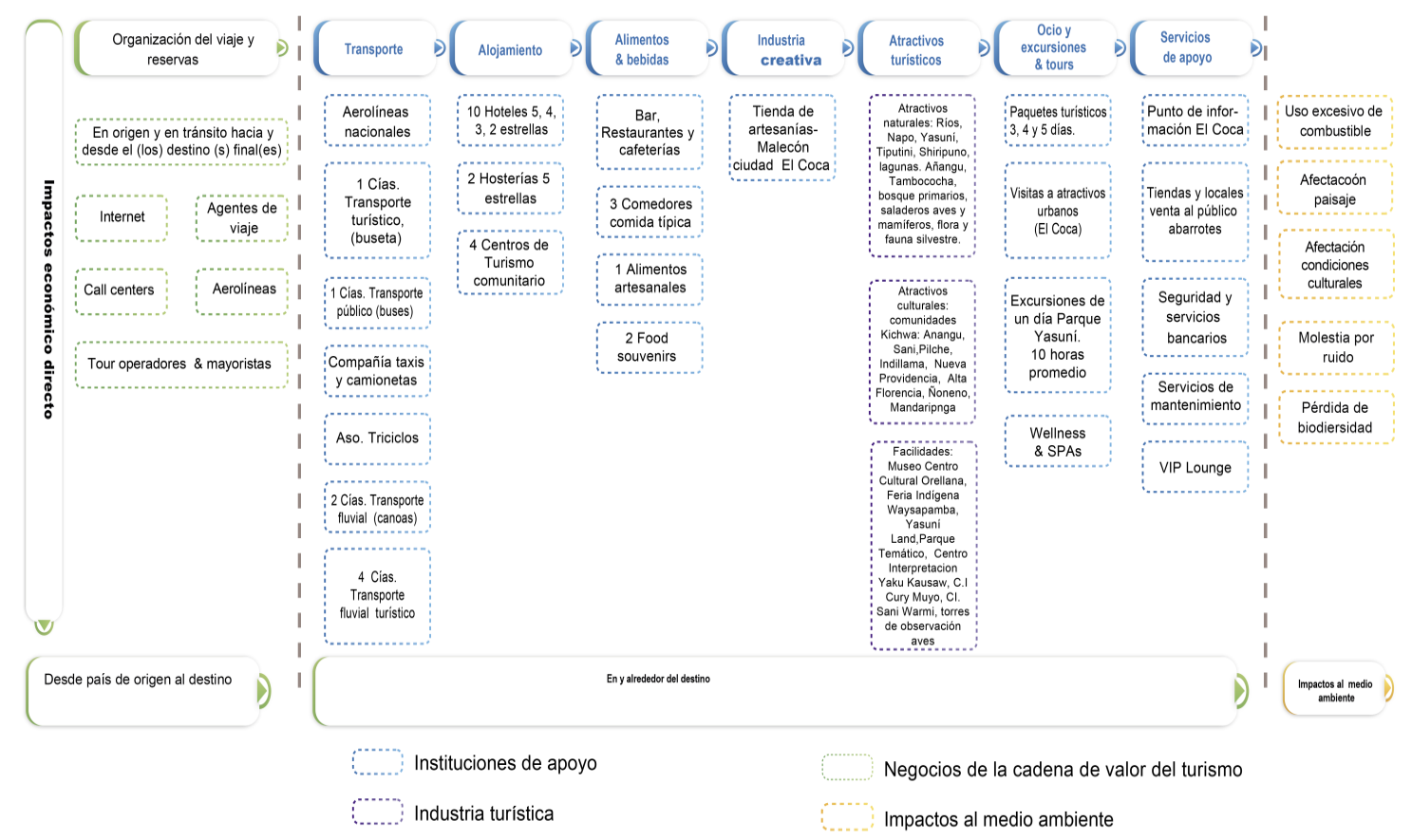

Figura. 6 Mapa de la Cadena de Valor de Turismo Parque Nacional Yasuní.

Fuente: United Nations Enviroment Programm UN Enviroment-Mapping the value chain, 2019.

\section{Discusión.}

El enfoque de cadenas de valor se ha aplicado inicialmente al ámbito del turismo para impulsar el desarrollo empresarial y, de manera más general, como herramienta para la planificación estratégica con la finalidad de mejorar la competitividad. Otras metodologías se concentran en identificar las sinergias y articulaciones existentes en un 
conjunto de actores de un subsector productivo que se localizan en un espacio geográfico determinado, donde el objeto principal del análisis es generar recomendaciones para fortalecer la red de actores en torno a la generación de un bien o servicio que garantice el desarrollo económico a las comunidades. (Coppin Leave. 2009).

Desde la perspectiva de esta investigación y como referencia sobre la metodología planteada en torno a la identificación de un subsector, se consideró un área protegida como un espacio geográfico con vocación turística y que ha sido poco estudiado en torno a la incidencia de la actividad turística perse, menos aun desde la perspectiva de cadena de valor. En el contexto macro, la Vicepresidencia de la República del Ecuador (2013) presentó algunos factores que define la cadena productiva, el estudio refleja indicadores para el dimensionamiento de la cadena de turismo sobre las actividades de impacto económico indirecto o soporte, y realiza un corto análisis de las actividades de impacto económico directo o primarias que componen la cadena de valor. Otros destinos han buscado desarrollo sectorial en el entorno local a través del fomento de la cadena aplicado a ciertos productos turísticos como Turismo de Naturaleza, Aventura, Aviturismo. En el contexto empresarial se ha evidenciado estudios específicos a nivel estratégico que permiten consolidar las teorías expuestas para mejorar la competitividad.

La Cadena de Valor en Turismo es un concepto poco utilizado por los gestores y prestadores de servicio, y en áreas protegidas quizá desconocido, aunque se puede crear una relación constructiva con el sector turístico, trabajan en contextos jurídicos, políticos, económicos y culturales que restringen en gran medida su libertad. La gran importancia documental, mediática, científica, cultural, turística y medio ambiental que se le ha otorgado al Parque Nacional Yasuní en varios momentos y contextos nacionales e internacionales propiciaron a tomar este espacio para la identificación, mapeo y análisis de la incidencia de elementos de la cadena de valor turismo, ligado a que aquellos gestores que intervienen en el contexto turístico y las relaciones entre los mismos; posiblemente puedan tener una guía sobre dónde intervenir, su viabilidad y efectividad.

\section{Conclusiones.}

- La identificación y análisis de los gestores involucrados en la operación de la actividad turística del Parque Nacional Yasuní junto con el tratamiento estadístico de 32 variables estudiadas permitió definir la cadena de valor del área protegida y los eslabones que generan impactos económicos directos en relación a las actividades primarias de la cadena que, resultan estratégicas para el rendimiento y toma de decisiones más asertivas para el sector.

- El instrumento de recopilación fue validado por Alpha de Conbach a través del procesador estadístico IBM SPSS Statics 22, que determinó un alto grado de fiabilidad de los ítems propuestos, en el análisis estadístico 2 variables no presentaron variabilidad, la desviación estándar igual a 0 omitieron su uso para 
el cálculo de los componentes principales. El análisis de suficiencia KMO al ser superior a 0,5 reflejó la pertinencia de realizar análisis de componentes, finalmente con el $63,89 \%$ de varianza total explica el sistema con cuatro componentes y nuevas variables.

- Asimilar la complejidad y transversalidad del turismo como actividad económica con un enfoque de cadena de valor representa una visión pertinente sobre las interrelaciones en los sistemas de valor que confluyen en el Parque Nacional Yasuní. El fortalecimiento de la red de actores que se encuentran identificados y mapeados en la cadena podría garantizar el desarrollo económico de las comunidades involucradas y la meta de sostenibilidad que busca el área protegida.

\section{Referencias bibliográficas.}

Alliance, Rain Forest. (2005). Guía de Buenas Prácticas de Turismo Sostenible para Comunidades de Latinoamérica Una guía para iniciativas turísticas comunitarias. Epicentro Publicidad. San José de Costa Rica.

Calderón, Á. (2015). Análisis de la Cadena del Turismo. Comisión Económica Para América Latina y El Caribe CEPAL. Quito Ecuador. Recuperado de http://www. vicepresidencia. gob. ec/wp content/uploads/2015/07/Resumen Cadena Turismo

Cisneros, P. (2007). Gobernanza ambiental y conservación de la naturaleza, un caso de control territorial indígena en el Parque Nacional Yasuní (Master's thesis, Quito: FLACSO sede Ecuador).

Coppin, L. (2009). Cadenas de Valor en turismo.

Christian, M., Fernandez-Stark, K., Ahmed, G., \& Gereffi, G. (2011). The tourism global value chain. Center on Globalization, Governance \& Competitiveness $(C G G C)$, Duke University.

Gastélum-Escalante, J., Patiño, D. C., González, B. Z., Cárdenas, F. P., Narváez, Y. V., Sierra, V. P., . \& Pinochet, G. V. Notas del Editor. La cadena de valor del turismo, un abordaje teórico-conceptual en algunos países de Iberoamérica y el Caribe Page 9. Ciencia desde el Occidente, Vol. 5 | Núm. 1 | Diciembre de 2018

Ministerio del Ambiente. Programa- Reparación Ambiental y Social (PRAS), (2016). Resultados de la Implementación del Plan de Manejo de Visitantes del Parque Nacional Yasuní.

Ministerio del Ambiente del Ecuador.(2011) Plan de Manejo Parque Nacional Yasuní.

Ministerio de Turismo del Ecuador. (2019). Plan Nacional de Turismo 2030. 
Rahmiati, F., Ismail, Y., Amin, G., Simatupang, T., Larso, D., \& Othman, N. A. (2019, September). Tourism Value Chain Activities Model for Competitive Advantage Measurement. In International Conference on Trade 2019 (ICOT 2019). Atlantis Press.

Secretariat of the Convention on Biological Diversity (2015) Tourism Supporting Biodiversity- A Manual on applying the CBD Guidelines on Biodiversity on Tourism Development, Montreal, 56 pp.

Subsecretaría de Turismo, 2015. Plan de Acción de Turismo Sustentable en Áreas Protegidas del Estado 2014-2018. Santiago, Chile.

Springer-Heinze, A., Albert, H., Bagwitz, D., Banjara, G. B., Bertenbreiter, W., Burk, C., ... \& Fleischer, G. (2007). Value Links Manual.". The methodology of value chain promotion, First Edition. GTZ, 221.

World Tourism Organization (2019), UNWTO Tourism Definitions, UNWTO, Madrid.

TurisConsulting, I. T. C. Ltda.(2016) Libro 1-Plan Estratégico de Desarrollo Turístico y Mercadeo del destino Cuenca y su área de Influencia 2016-2021 (Book 1Strategic plan for tourism development and marketing of the destination Cuenca and its area of influence 2016-2021). Cuenca, Ecuador: Fundación Municipal Turismo para Cuenca, 115.

Tourism and Trade: A Global Agenda for Sustainable Development. Geneva: ITC/UNWTO,2015. xi, 36 pages.Doc. No. SC-15-335.E

\section{LCiencia}




\section{PARA CITAR EL ARTÍCULO INDEXADO.}

Valdez Quinte, S. X., Segura Chávez, E. O., Cabanilla Vásconez, E. A., \& Ruiz Mármol, E. E. (2021). Elementos de la cadena de valor que inciden en el desarrollo de la actividad turística del Parque Nacional Yasuní. ConcienciaDigital, 4(2.2), 120-138. https://doi.org/10.33262/concienciadigital.v4i2.2.1782

\section{LCiencia}

El artículo que se publica es de exclusiva responsabilidad de los autores y no necesariamente reflejan el pensamiento de la Revista Conciencia Digital.

El artículo queda en propiedad de la revista y, por tanto, su publicación parcial y/o total en otro medio tiene que ser autorizado por el director de la Revista Conciencia Digital.

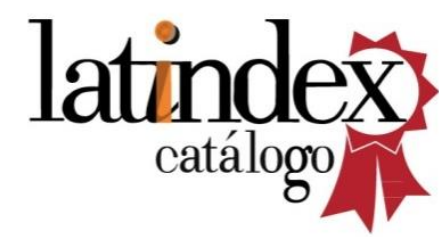

